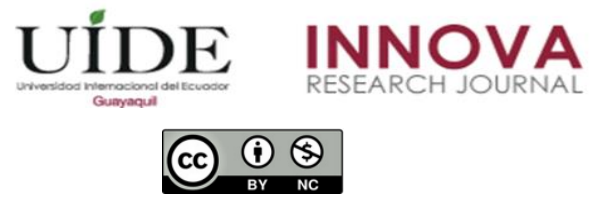

INNOVA Research Journal, ISSN 2477-9024

(Septiembre-Diciembre 2019). Vol. 4, No.3.1 pp. 150-167

DOI: https://doi.org/10.33890/innova.v4.n3.1.2019.1092

URL: http://revistas.uide.edu.ec/index.php/innova/index

Correo: innova@uide.edu.ec

\title{
Naturaleza ontológica de la investigación socioeducativa: Elementos orientadores
}

\section{Ontological nature of socio-educational research: orienting elements}

\author{
Alejandra Mercedes Colina Vargas \\ Universidad ECOTEC, Ecuador \\ https://orcid.org/0000-0003-1514-8852
}

Autor para correspondencia: acolina@ecotec.edu.ec

Fecha de recepción: 15 de julio de 2019 - Fecha de aceptación: 17 de octubre de 2019

\section{Resumen}

El presente artículo propone la exploración y revisión bibliográfica de forma narrativa de las categorías fundamentales que conforman la investigación socioeducativa, concebida ésta última como un fenómeno social, delineando con ello el ámbito epistemológico del fenómeno educativo, que emerge de la simbiosis entre la naturaleza de la realidad y las características que las rodean. Para llevar a cabo el análisis teórico se realizó una búsqueda en bases de datos científicas, haciendo énfasis identificar los aspectos filosóficos, científicos y técnicos que delinean las relaciones entre elementos epistémicos que conforman la investigación socioeducativa, los cuales se resume finalmente en un perfil de diseño de investigación de los fenómenos socioeducativos. Dentro de las reflexiones finales destaca el hecho de la existencia de vacíos al emprender una investigación socioeducativa, pues se intenta la producción y reproducción del proceso de investigación social de manera lineal, sin tomar en cuenta las relaciones analíticas, hermenéuticas y reflexivas que circundan sobre los fenómenos educativos estudiados con perspectiva científica.

Palabras claves: investigación educativa; proceso de investigación; naturaleza ontológica; realidad socioeducativa

\begin{abstract}
This article proposes the exploration and bibliographic review in a narrative way of the fundamental categories that make up socio-educational research, conceived as a social phenomenon, thereby delineating the epistemological scope of the educational phenomenon, which emerges from the symbiosis between the nature of the reality and the characteristics that surround them. To carry out the theoretical analysis, a search was conducted in scientific databases, emphasizing the identification of philosophical, scientific and technical aspects that delineate the relationships between epistemic elements that make up socio-educational research, which are finally summarized in a design profile of investigation of socioeducational phenomena. Among the final reflections, the fact that there are gaps in undertaking socio-educational research stands out, since the production and reproduction of the social research process is attempted in a linear manner, without taking into account the analytical, hermeneutical and reflexive relationships that surround the Educational phenomena studied with a scientific perspective.
\end{abstract}

Key words: educational research; research process; ontological nature; socio-educational reality 


\section{Introducción}

Los rasgos característicos del mundo actual, requieren que la sociedad impulse el progreso de la ciencia, la educación y la cultura en contextos socioeducativos dinámicos y complejos, para transformar escenarios que permitan constituir individuos integrales, críticos, conscientes, pensantes y atraídos por la realidad que los envuelve, con la finalidad de resolver los problemas que se presenten en la cotidianidad. En este sentido, toda investigación, indiferentemente del enfoque que se utilice, toma como punto de partida una idea o una inquietud particular, por parte de quien investiga. Por ello, el abordaje inicial de una realidad socioeducativa, surge precisamente cuando se consideran los conocimientos adquiridos, la experiencia profesional, y el trabajo cotidiano, permitiendo la identificación e interrelación de variables o categorías inmersas en ese contexto.

Siendo el caso que ocupa en este artículo, el proceso de investigación en el área educativa, posee una lógica propia que da sentido al trabajo de indagación sobre la realidad social y socioeducativa que se desee investigar, partiendo de la premisa que la educación constituye un fenómeno social, el cual requiere de un esfuerzo y definición de los límites de la explicación y el conocimiento desde el ámbito epistemológico del fenómeno educativo en sí, así como, la validez del conocimiento que se construye sobre esta disciplina.

Tomando las consideraciones anteriores como base, este articulo pretende dilucidar atributos conceptuales relacionados con la naturaleza epistémica de la investigación socioeducativa, para lo cual se describen cada uno de los elementos que la componen, tales como el estado del arte, la naturaleza ontológica, la situación problemática, la intención del estudio y el escenario o contex to social, asumiendo las visiones de diferentes autores. No pretendiendo describir cómo afrontar problemas educativos, ni tampoco delinear una receta de componentes rígidos que conformen el proceso investigativo, más bien perfilando pinceladas en torno a los elementos que pudieran ser imprescindibles al momento de emprender un trabajo investigativo desde cualquier enfoque epistemológico.

Se plantea entonces, iniciar el desafío a partir de la recopilación de piezas relevantes de información obtenido de diferentes fuentes que tributen en el abordaje de dicha investigación con el propósito de intentar descubrir vacíos de conocimiento existentes en la realidad, que tome en consideración una serie de acciones humanas racionales, planeadas en función de afinar y estructurar lógicamente las ideas en el matiz socioeducativo, con base en hechos u objetos inmersos en los procesos investigativos.

\section{Métodos}

La metodología empleada en la presente investigación toma en cuenta elementos de la investigación documental recogidos de bases de datos científicas a partir de fuentes avaladas por la academia como son: libros, revistas científicas, trabajos de investigación, artículos. Se ubica dentro de un estudio cualitativo de corte descriptivo, exploratorio y no experimental. Se aplicaron métodos teóricos de carácter inductivo -deductivo donde emergen reflexiones y análisis crítico de los elementos que conforman la naturaleza ontológica de una investigación educativa a partir de la información más destacada. 


\section{Marco teórico}

\section{Estado del Arte}

Cuando se aspira realizar una investigación, sea cual sea su naturaleza científica, se parte de conocer lo que se ha hecho en el pasado, de una re-construcción relacionada al tema a tratar, es decir corresponde al punto de partida, el principio de todo proceso de investigación, por su parte el filósofo griego Aristóteles, determinaba el principio desde varios puntos de vista, siendo uno de ellos, cuando hace referencia "al punto de una cosa desde donde alguien puede comenzar a moverse", otra perspectiva se concibe como "el punto desde donde cada cosa puede hacerse del mejor modo" y finalmente como "aquello desde lo cual, sin ser intrínseco a la cosa, ésta comienza a hacerse y desde donde principian naturalmente el movimiento y el cambio".

Contextualizando, lo señalado por el filósofo, se expresa al estado del arte como el punto de partida donde el investigador debe comenzar a moverse, para hacer mejor las cosas, tomando en consideración la evolución, desarrollo y cambios del objeto de estudio desde sus inicios hasta el presente. Para ello es necesario iniciar este apartado con una gran interrogante ¿Cuál es el origen del estado del arte?, la respuesta es manifestada por la primera persona que realizó un estado del arte sobre el mundo, Aristóteles a través de su gran obra emblemática titulada Metafísica, en la cual determina los inicios de la ciencia a través del conocimiento, y este último por la curiosidad innata del ser humano, estableciendo así, una distinción y articulación entre ciencia, arte y experiencia.

De lo anteriormente expuesto surge una segunda interrogante ¿Qué se entiende por estado del arte?, el significado de la expresión ha ido evolucionando desde el siglo XX hasta el presente, significaba la etapa en que se desarrollaba una disciplina práctica o tecnología, hoy en día, uno de los significados vigentes se encuentra en la controversia de las más nuevas o mejores técnicas de un producto o actividad (Carramolino, 2009, p. 1).

El estado del arte es referido como el estado en que se encuentra el problema de investigación comprende el punto inicial de indagación en el proceso investigativo, realizando una revisión exhaustiva sobre las fuentes bibliográficas, investigaciones, textos, artículos o ensayos, tesis doctorales, conferencias, centros de investigación que han tratado el tema de investigación, así como también las experiencias que tienen relación con el problema (Toro y Parra, 2006).

De igual manera, Nieto (2008), establece que el estado del arte como el análisis sistemático y valorativo del conocimiento, considerando además las producciones que giran en torno al campo de investigación científica, en un determinado periodo de tiempo. Por su parte, Jiménez y Cols.(2006), conciben el estado de arte como producto de lo actual, reconociendo lógicas investigativas antepuestas al tema que se pretende desarrollar y que, mediante distintos abordajes y metodologías, se han llegado a reflexiones y opiniones diversas, ineludibles de consultar, convirtiéndose así en una obligación investigativa inspeccionar dichos acumulados.

Transcurriendo en el abordaje sobre el estado del arte, se pretende dar respuesta a ¿Por qué es de utilidad el estado del arte en un proceso de investigación?, Arellano y Santoyo (2009), 
señalan que el estado del arte es de suma importancia, porque contextualiza el problema investigativo en la contemporaneidad científica con conocimientos anteriores a él, permitiendo percibir las perspectivas de descubrimientos científicos y los conocimientos inéditos, buscando situarnos a la vanguardia del conocimiento actual.

El estado del arte, establece la necesidad de hacer un alto en la investigación, teniendo como apoyo una determinación temporal, el cual permitirá fluctuar la investigación con el propósito de consolidar la información que se tiene a la mano, para de alguna manera decantar y cuestionar, posteriormente llegar a un conocimiento de la temática establecida. De igual forma, permite la evolución del conocimiento e impide consumir esfuerzos y tiempo en compilar información innecesaria, es decir, que ya existe, o información errónea proveniente de estudios anteriores.

Desde la primera visión, como abordaje de un problema, se está planteando una necesidad de interpretar los corpus teóricos, donde lo que se busca con ello es el conocimiento y la apropiación de la naturaleza que persigue la realidad social del objeto de estudio. Una segunda perspectiva, como producto de lo dado, se puede presentar como resultado de una investigación, estableciendo los marcos referenciales de cualquier tipo de investigación, puesto que lo que evidentemente busca el investigador en la información leída que le antecede al objeto de estudio, es armar el rompecabezas conceptual que le permita al investigador, interpretar la realidad y comprenderla desde un sentido común de trascendencia.

Otro sentido, se vislumbra, como punto de partida a lo inédito, permitiendo no solo conocer la problemática y problematizarla todavía más, sino establecer rutas vírgenes, donde nadie ha transitado metodológica o conceptualmente, por lo que no parten de la nada sino de lo acumulado históricamente. De alguna manera, el sujeto llega a apropiarse del conocimiento contemporáneo, para posteriormente proyectarse en lo inesperado. Se requiere unificar criterios en torno al estado de arte para el proceso de investigación cualitativa, como guía para la formulación y ejecución de proyectos e incluso en investigaciones de mayor envergadura (Gómez, Galeano y Jaramillo, 2015).

Finalmente, el estado de arte establece la re-construcción crítica del objeto de estudio esbozado a partir de un diálogo de saberes, como una búsqueda constante de la voz de los investigadores, participantes y de los contextos sociales y políticos. A partir de la construcción de estrategias metodológicas que promueven la participación, la autocrítica y la deliberación de las comunidades educativas intervinientes (Guevara, 2016).

\section{Propósito de la investigación}

En este apartado se puntualizan las acepciones de ciertos términos que correspondan al contexto de estudio como son: objetivos y propósitos. Partiendo entonces del término, propósito, el mismo proviene del latín propositum compuesto del prefijo pro (hacia adelante) y positum (ponere, participio de poner) es decir consiste en la intención de hacer algo o dejar de hacer algo.

Una vez establecido el estado del arte del tema de investigación de acuerdo a lo señalado por Gurdián (2007) se debe proceder a definir por parte de los investigadores, la aspiración, el 
para qué, la intención, es decir, el propósito de desarrollar la investigación que presupone al objeto transformado, o sea, la situación propia del problema superado.

Por su parte, para Creswell (citado por Rodríguez G. y Valldeoriola R., 2009):

la declaración del propósito establece la dirección para la investigación. De hecho, la declaración del propósito es el comunicado más importante de una investigación, y desde éste, se desarrollan el resto de aspectos de la investigación. (Creswell, 2003, p. 87).

En relación a lo antes señalado, estos propósitos establecen la dirección central del estudio a través de enunciados sobre las implicaciones, alcances, aportes, proyección y/o utilidad que se espera de los resultados de la investigación (académicos, sociales, políticos, prácticos, metodológicos, teóricos, personales)

De igual manera, Campos y Villalobos (Citado por De Melo y Casanoves, 2010) destacan que los propósitos pueden ser: indagativos, prácticos y personales. Estos propósitos difieren tanto para las investigaciones cualitativas, cuantitativas y mixtas. Se tiene entonces que, dependiendo del fin de la investigación cuantitativa se emplean variables: independientes, dependientes, intervinientes, los verbos usados: explicar, comparar, describir, estimar, evaluar. Por su parte, los propósitos de las investigaciones cualitativas, la intencionalidad se centra en la descripción, exploración, interpretación, transformación, se recomienda el uso de verbos como, por ejemplo: caracterizar, describir, examinar, explorar, comprender, descubrir, transformar.

\section{Naturaleza Ontológica de la realidad socio-educativa}

La naturaleza ontológica de la realidad socio-educativa, corresponde a una de las primeras interrogantes que debe generarse un investigador, puesto que supone el establecimiento de los principios y fundamentos del conocimiento sobre una realidad o fenómeno, según la óptica de éste, en base a lo que estudia, por lo que esta pregunta ontológica, puede plantearse en términos de ¿Cuál es la forma y la naturaleza de la realidad, más aún, ¿qué es aquello que podemos conocer de ella? Por consiguiente, para esclarecer este cuestionamiento, se abordarán algunos términos vinculantes, como naturaleza, realidad, ontología, entre otros, considerando los diferentes enfoques de investigación asumidos por el investigador.

Es así como, la naturaleza, según la perspectiva aristotélica, es concebida en muchos sentidos; a) como aquello primero o inmanente a partir de lo cual crece lo que crece, b) como el elemento primero e inmutable desde su propia potencia, del cual es o se hace alguno de los entes naturales, y c) la substancia de los entes naturales, que a su vez están constituidos por la especia y la forma. De allí, que la naturaleza es considerada como lo primero, el elemento y la substancia para dar respuesta a las incógnitas que puede plantearse el sujeto que investiga sobre lo que investiga.

Puntualizando ahora el término ontología, puede referenciarse lo planteado por Rodríguez \& Valldeoriola (2009), como la disciplina que se preocupa por la cuestión del ser. El término ontología comienza con los postulados de Aristóteles, en tanto que se relaciona con la metafísica y surge a comienzos del siglo XVII, siendo designada como el estudio de todas las cuestiones 
que afectan al conocimiento de los géneros supremos de las cosas y tiene como misión la determinación de aquello en lo cual, los entes consisten y aun de aquello en que consiste, el ser en sí (Ferrater, 2008).

Si bien se ha hecho notable, lo que el investigador considera como naturaleza o principio de lo que estudia, al mismo tiempo que se ha especificado la ontología como el estudio de los entes y del ser en sí, es importante destacar lo que percibe, y lo que puede reconocer como elemento o dimensión de su realidad, para dar explicación a lo que investiga.

En este hilo discursivo, lo real, puede entenderse como lo que "es" o "existe", por lo que la idea de lo que es real, tiende a asemejar la realidad con lo dado en el marco de la experiencia (de la sensación). La realidad es una de las maneras primarias del ser, y puede distinguirse como uno de los momentos del ser (en función de su existencia), como una de las formas del ser (en oposición a la idealidad) y como uno de los modos del ser (con respecto a la actualidad). En base a lo anteriormente planteado, es imperante que en una ontología crítico descriptiva, se establezcan claramente estas distinciones de lo que es real, evitando así, aplicar a una forma de realidad, categorías que no correspondan a ella (Ferrater Op. Cit.).

Continuando con el desarrollo del artículo, Maturana (1997) expresa que la realidad es entendida como una proposición explicativa, en tanto que, no es una experiencia, sino más bien, un argumento dentro de una explicación Dicha proposición, se relaciona directamente con la experiencia de las coherencias operativas de la vida cotidiana y técnica de un individuo; por lo cual, esta realidad surge en función de si el investigador que observa, acepta o rechaza la teoría sobre el origen biológico de sus propiedades como tales.

Sobre este silogismo, Maturana (op. cit) establece dos dominios ontológicos como formas fundamentales que un observador puede adoptar, dependiendo de si demanda o no una línea explicativa biológica, para dar respuesta a lo que percibe en su ambiente y en su contexto, por lo que, dichos dominios los clasifica en relación a las ontologías trascendentales y las ontologías constitutivas.

Describiendo detalladamente esta clasificación, se tiene que, cuando un observador se encuentra en la línea explicativa de las ontologías transcendentales (sin paréntesis), éste acepta a priori, como fuente de validación de sus explicaciones, una realidad objetiva que es independiente y externa a él. En consecuencia, el observador dentro de esta línea explicativa, ve la realidad como lo es, no como una proposición explicativa, por tanto, ésta es simple, reductible y fragmentable.

En oposición a lo anterior, cuando un observador se encuentra en la línea explicativa de las ontologías constitutivas (entre paréntesis), éste acepta que la realidad es lo que él hace al validar sus explicaciones dentro de su cotidianidad, y al hacerlo, producen tantos diferentes dominios de realidad como diferentes dominios de entidades que están constituidos en su acto de explicar; por lo que el observador se percata de distinguir cada dominio de su realidad en base a las coherencias operacionales de su praxis de vivir. En consecuencia, dentro de esta línea explicativa, la realidad es multidiversa, construida y holística. 
Considerando todos los argumentos anteriores, los supuestos ontológicos en una investigación, cubren ideas generadoras, en tanto ¿Cómo se concibe la naturaleza de la realidad?, ¿Cuál es la creencia que mantiene el investigador con respecto a la realidad que investiga?, por lo que las respuestas a estas interrogantes, pueden ser comprendidas desde la visión de mundo o perspectivas concebidas por dicho investigador, tal como señala Gurdian (2007).

En este sentido, apoyando los planteamientos de Sandoval (1996), Guba y Lincoln (citados en Denman y Haro, 2002) los positivistas observan en su realidad, las entidades o cosas, que pueden ser conocidas mediante generalizaciones relativamente separadas del tiempo y del contexto, bajo la forma de leyes causales de carácter absoluto. Por consiguiente, los investigadores que se suman a este enfoque (realismo ingenuo), sostienen que sólo existe una sola realidad (Universo) y buscan dar explicación de ella, constituyendo una cadena causaefectos, que dará cuenta de un hecho social independiente de otro hecho social, es decir, exterior a los individuos. Sin embargo, los post-positivistas a diferencia de los positivistas, se inscriben en un realismo crítico, asumiendo que existe una única realidad, pero es imperfectamente aprehensible, ya que su obediencia a leyes naturales, sólo podrá ser entendida de forma incompleta, puesto que la naturaleza de los fenómenos es esencialmente incontrolable.

Ahora bien, haciendo referencia a los investigadores que se adhieren al enfoque cualitativo, circunscribiendo sus diferentes corrientes (interpretativo, socio-crítico y pragmático), Mella (2007), sostiene que en este enfoque, la realidad es contextualizada y prácticamente inseparable del tema del holismo, siendo éste último, concebido por Barrera (2008) como la apreciación de un evento en su mayor complejidad y en su contexto para obtener una visión completa y amplia de lo que se estudia, sin agotar ni la comprensión ni el evento.

En este sentido, las implicancias del contextualismo y del holismo, en un enfoque investigativo de carácter cualitativo (sea este naturalista, transformativo, emergente), engendran según Mella (2007), un estilo en el cual, los significados que se le dan a las conductas tanto individuales como colectivas de las personas, tienen que ser puestos en el contexto de los valores, prácticas y subsecuentes estructuras de la entidad apropiada. Es así como, dentro de este enfoque, la realidad es asumida en la acción misma donde ella se produce, más allá de las causas y de los efectos observables, pero sin que éstos, a su vez, sean descuidados por el investigador.

Por consiguiente, los investigadores cualitativos según Prévost y Roy (2007), buscan fijar el conocimiento en la comprensión de categorías y procesos para dar explicación al mundo que los rodea, y para ello, presumen una posible extrapolación de sus descubrimientos más allá del objeto de estudio, construyendo procesos emergentes para dar sentido a su realidad.

Sobre las consideraciones antes referenciadas, es importante señalar, que, dentro de la investigación cualitativa, la frontera entre lo interno y lo externo se convierte en ilusión, en el sentido de que el investigador amplía su consciencia para abordar el fenómeno que estudia y se ubica en la consciencia del otro (ponerse en el zapato del otro), experimentando procesos de interconexión y cambio en el flujo de los acontecimientos que se suscitan en la realidad que investiga. 
Como puede observarse, lo ya argumentado, coincide con la clasificación de los dominios ontológicos plasmada por Maturana, donde no existe una sola realidad, sino un multiverso de realidades interrelacionadas e interdependientes, por lo que, dentro de la óptica cualitativa, los elementos éticos, políticos y epistemológicos de los que dispone el investigador, exigen que la realidad sea colocada entre paréntesis (ontología constitutiva), para reconocer la naturaleza elusiva de lo real, su indeterminación y su complejidad, ya que no es una condición dada sólo por el sujeto investigador, sino también por la propia naturaleza del contexto.

A lo anterior, puede adosarse lo plasmado por Mella (2007), quien expresa que en la investigación cualitativa, existen dos aspectos importantes, estos son la introspección y la empatía, por tanto, el primero de ellos, implica entender las impresiones propias de quien investiga, lo que genera un acercamiento a sus pensamientos y sentimientos, dando significado a un accionar, aunque dichas acciones, sólo tienen sentido en base a la experiencia del sujeto, en el intento de entender los acontecimientos de su realidad. El segundo aspecto, es la empatía, que implica la aceptación del otro, como un legítimo otro, respetando y valorando sus ideas y opiniones.

Estos dos aspectos, juegan un papel decisivo en la investigación cualitativa, puesto que permiten conocer y comprender un mundo e interpretar una realidad, construyendo relaciones entre sujeto-sujeto, sujeto-objeto y sujeto-contexto, lo que hace, que, a su vez, la realidad dependa de un sujeto cognoscente que concibe distintas formas de percibir, pensar, sentir y actuar. La realidad es creada, cambiante, multidiversa y holística, que se mueve en un sistema circular que conlleva al entendimiento de la vida, como un proceso de conocimiento basado en acontecer y la experiencia en congruencia con un medio. De allí que, tanto el investigador como la realidad emergen como explicaciones de las experiencias del mismo y no como entidades trascendentales.

Develando con ello entonces, la incorporación del vacío en la ontología de la educación representa una ofensa a la supremacía de todo acto educativo que pretende responder a la transferencia evitando el carácter de casualidad que infringen contra las "planificaciones" y que, en muchas veces, son traducidas como "indisciplina" o "desafío a la autoridad" (Collela, 2016, p. 171).

Atendiendo a todo lo anteriormente planteado, la realidad socio-educativa debe ser comprendida como una realidad sujeta a cambios, con rupturas y distinciones, lo que constituye un espacio para la construcción de nuevos saberes científicos y populares, partiendo de la interpretación de dicha realidad en su propio terreno, registrando simbolismos, categorías y significados, al mismo tiempo, que se entreteje una red de relaciones conceptuales y conflictivas que dan sentido a las acciones del investigador.

Se requiere entonces, de renovadas concepciones epistemológicas que involucren una realidad que recupere la esencia humana y considere la pluralidad de culturas, pues como señala Gadamer (1995), el hombre se entiende a partir de su entorno y su vida cotidiana, y se articula con éste en la forma del lenguaje en el que se mueve, generando siempre un ocultamiento y un desocultamiento en su estructura de pensamiento. 
El ser humano se gesta como un ser que hace cultura en un acontecer histórico, y es a partir de su historicidad, que le resulta posible comprender el fenómeno de la realidad cultural socio-educativa. Esto conlleva, a que el ser humano, en su práctica investigativa no pueda disociar el conocimiento del acontecer y las relaciones de la vida, para interpretar y buscar explicaciones de las condiciones bio-antropo-socio-culturales emergentes en la realidad que se aborda.

\section{Precisando la situación problemática}

Considerando el contexto antes presentado, es indudable que una investigación se origina a partir de ideas, y que éstas últimas, constituyen el primer acercamiento a la realidad que habrá de investigarse. Dentro de la cantidad de fuentes que pueden generar ideas de investigación, se pueden tener las experiencias vividas, reuniones en grupos, materiales, revistas, libros, referentes televisivos o recordar hechos pasados.

Es de esta manera como, las ideas iniciales, deben analizarse cuidadosamente para ser adaptadas en planteamientos con detalles precisos y sistemáticos. Por tanto, se hace necesario familiarizarse con el área de conocimiento de la idea de investigación, reflexionando sobre el enfoque que ha de adentrarse en el objeto de estudio, lo que conviene leer y revisar producciones con acercamientos al contexto de esgrimir ideas problematizadoras. Atendiendo a ello, se hace menester detenerse para especificar, desde una perspectiva general, las acepciones de "problema" establecidas por varios autores.

En tal sentido, Bunge (1972), designa la palabra problema como cualquier dificultad que no puede resolverse automáticamente, es decir, con la sola acción de reflejos instintivos incondicionados, o mediante el recuerdo de lo aprendido anteriormente. De igual forma, Palella y Martins (2004), expresan que un problema "es para un actor, la formalización de una discrepancia entre la realidad constatada y una norma que él acepta o crea como referencia". (p.40)

Por otro lado, Padrón (1996), establece que el sentido pragmático del término problema, surge por los cambios que se generan en un entorno estando teleológicamente orientados para alcanzar un fin, a través de una serie de acciones (no siempre racionales) que influyen en la selección de pasos estratégicos para transitar de una situación a otra. De igual manera, refiere que un problema se encuentra estrechamente vinculado a la necesidad de aplicar "cuerpos informacionales disponibles y ya consolidados para la transformación de dificultades en soluciones". Es decir, cada vez que se plantea una finalidad que origine un cambio, es porque la situación actual resulta problemática o por lo menos puede llegar a ser mejorable.

Al respecto de las concepciones expuestas, se puede definir el problema de investigación como: situaciones o cambios que ocurren en una realidad, originando dificultades que afectan a un individuo y obligan ser satisfechas, por lo que éstas deben ser estudiadas a profundidad para la comprensión de las relaciones entre sus componentes y efectos, a objeto de aproximarse a posibles soluciones mediante una serie de acciones que permitan transformar dicha realidad. 
Siguiendo la dinámica del texto, para Gómez (2008), el problema es objetivo, en tanto refiere a una situación presente en el objeto, pero es también subjetivo, pues para que conste el problema, la situación tiene que generar una necesidad en el sujeto. Un mismo problema de investigación, puede admitir diferentes planteamientos y distintas soluciones, según el punto de vista conceptual desde el cual se lo analice y defina.

Asimismo, para Bunge (1972), un problema puede considerarse planteado científicamente, si se formula en términos inteligibles y precisos, disponiendo además de un fondo de conocimientos en el que pueda encajar, y puede abordárselo mediante algún procedimiento (método o técnica). Es así como, en la realidad a investigar se ubican un conjunto de datos susceptibles de capturar para referir posibles soluciones y que éstas pueden venir determinadas a partir de intereses teóricos de confirmación o refutación de supuestos.

Con base a dichas condiciones, antecedentes y matiz de referencia de otros trabajos, se puede delinear, plantear o formular el problema de investigación, en palabras concretas, representa estructurar formalmente el tema que se va a investigar, explicitando lo susceptible a ser investigado, y además, expresando la o las relaciones y posibilidades de observar la realidad. Al respecto, es importante precisar preguntas de investigación que resuman y condensen la esencia del problema, estableciendo parámetros temporales y espaciales del estudio para aproximarse a la presencia de las unidades de observación.

De hecho, la formulación de un problema, puede visualizarse desde la perspectiva cuantitativa o cualitativa. En base a la primera perspectiva Palella y Martins (2004), sostienen que "es importante partir de un planteamiento global, de una breve descripción del objeto de estudio, seguido por una serie de preguntas que ayuden a precisar lo que se pretende investigar" (p.47). Esto se logra, siguiendo una situación problemática a nivel macro o general, luego enunciarla a nivel meso, para finalmente a nivel micro, demostrar que el problema existe, haciéndola reflejo a través de evidencias objetivas, causas y consecuencias. Mientras que, desde la perspectiva cualitativa, según el planteamiento de Rueda, Ríos y Nieves (2007), la formulación de un problema, parte de ver la realidad desde diversos planos, lo que implica el planteamiento de aproximaciones sucesivas que permitan, en base a un conjunto de cualidades, recrear, reconstruir y repensar dicha realidad en un contexto lógico-dialéctico.

Siguiendo estas características sobre la precisión de afrontar una situación problemática de investigación, es menester aclarar que no siempre es sencillo formular los problemas de manera clara, precisa y manipulable, que refiere a la naturaleza de la investigación, de sus dificultades y complejas relaciones sistémicas. Es así como, en palabras de Rodríguez y Valldeoriola (2009), toda investigación sigue la fórmula dialógica del binomio preguntarespuesta, a cada tipo de pregunta (lo cual supone un "qué") le corresponde su propia respuesta; sin embargo, para formular correctamente una pregunta no asegura una buena respuesta, lo cual depende del método empleado para buscarla, pero es el primer paso para conseguirla.

\section{Justificación de la Investigación}

La justificación del estudio indica el por qué se quiere hacer esa investigación, expresa las razones tanto personales como generales del investigador; los aportes del estudio a 
determinada disciplina, a la solución de problemas prácticos de tipo organizacional, social, educativo, económico, cultural, entre otros.

En este sentido, se puede afirmar que consiste en describir las motivaciones por los cuales se lleva a cabo la investigación. A través de la justificación se resalta el por qué el problema es sustantivo y real, por qué se debe investigar y a quien a va a servir los resultados.

Por otro lado, Palella y Martins (2003) expresan que la justificación constituye el apartado del proyecto de investigación en la cual el investigador es libre de expresar y dar a conocer los fundamentos que lo mueven a realizar ese trabajo. Dando respuesta al ¿por qué es importante investigar la situación problematizadora?

De igual manera, al redactar la justificación se debe resaltar quienes serán los beneficiarios, en qué consiste y por qué es importante el desarrollo de la propuesta de investigación. En cada uno de estos aspectos se debe sustentar a través de las referencias temáticas de opiniones de diversos autores que apoyen la realización del diseño.

En líneas generales, la justificación debe responder a los siguientes momentos principalmente:

- Metodológico: se refiere al uso o propuesta de métodos y técnicas específicas que pueden servir de aporte y/o aplicación para otros investigadores que aborden problemas similares. ¿El resultado de la investigación ayudará realmente a resolver el problema? Con la investigación ¿Se creará nuevos instrumentos y técnicas de recolección de datos?

- Teórico: en este caso se pretende resaltar los supuestos que permitan avanzar en el conocimiento planteado o encontrar nuevas explicaciones que modifiquen el conocimiento inicial. En otras palabras, se hace énfasis en qué contribución o aporte tendría la investigación hacia otras áreas del conocimiento, tendría alguna importancia trascendental, los resultados podrán ser aplicables a otros fenómenos o ayudaría a explicar o entenderlos. ¿Los resultados de la investigación, complementan los postulados teóricos que la fundamentan?

- Practico: están destinados a resaltar la contribución a la solución de un problema concreto que afecta directa e indirectamente a una realidad social. Es decir, ayudaría a resolver algún problema presente o que surgiera en un futuro. ¿Ayudaría a resolver algún problema práctico?

- Relevancia Social: En que afectaría la investigación o que impacto tendría sobre la sociedad, quienes se beneficiarían con tal desarrollo. ¿Cuál es la relevancia socioeducativa? ¿Quiénes y de qué manera se beneficiarán?

\section{Intención: objetivos y propósitos}

En lo que se refiere a los objetivos de la investigación, una vez que se ha dilucidado los diferentes aspectos que comprende la enunciación de los propósitos y del problema, pueden ser formulado el problema como una o más preguntas, como objeto de indagación, o manera declarativa, como una o más hipótesis; posteriormente se declaran los objetivos o resultados que se aspiran en la investigación socioeducativa. 
Esta formulación de objetivos de la investigación, se realiza con la finalidad no de proyectar la solución científica exacta del problema, pero si contextualizar qué es lo que hay que hacer u obtener para satisfacer la necesidad planteada. En este sentido, Sabariego y Bisquerra (citado por Rodríguez G. y Valldeoriola R., 2009) destacan que "los objetivos de la investigación tienen la finalidad de señalar lo que se pretende y a lo que se aspira en la investigación." (p. 85)

Por su parte, Briones (1996) enfatiza que los objetivos de un proyecto de investigación expresan aquellos aspectos, relaciones, lógicas, dinámicas, efectos del problema, que pretenden ser esclarecidos en la investigación. En general, cristalizan la intención temática del investigador y corresponden a los aspectos por investigar provenientes del modelo de análisis, como las preguntas que quiere resolver el estudio.

Sin embargo, los objetivos pueden definirse como la expresión concreta de lo que se espera alcanzar no siendo ideales o abstracciones vagas, sino como el resultado práctico y tangible que se deben de alcanzar en cada etapa de la investigación.

Haciendo referencia a lo antes planteado, conviene destacar cuáles son las características o atributos de los objetivos tomando en cuenta lo planteado por los autores Legrá y Silva (sf.) y Palella y Martins (2003) presentados en la Ilustración 1. Generalmente, se distinguen dos tipos de objetivos: general (es) y específico (s). el primero señala lo que se pretende alcanzar con el estudio, dando respuesta a ¿Qué se quiere hacer? y ¿Qué se pretende obtener? Mientras que los específicos plantean una relación directa con el objetivo específico.

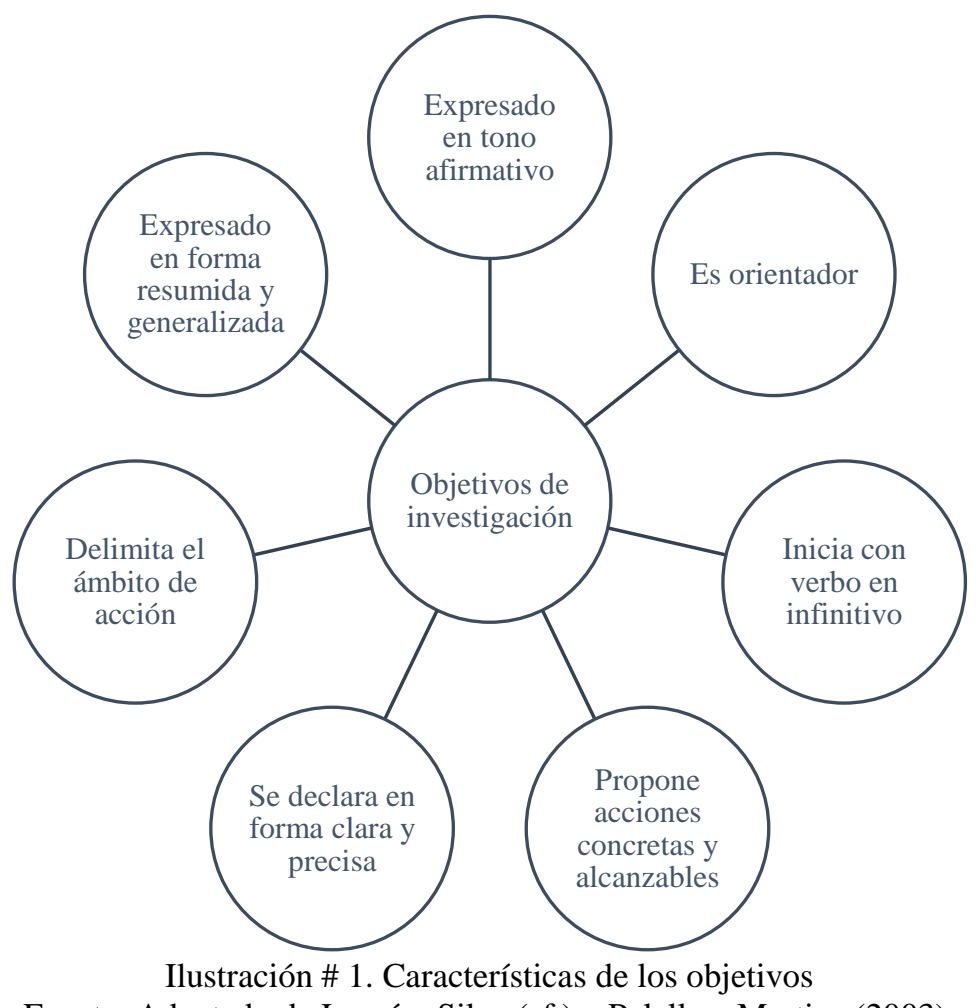

Fuente: Adaptado de Legrá y Silva (sf.) y Palella y Martins (2003). 
En otro contexto, haciendo alusión a la clasificación de los objetivos de investigación tomando en cuenta a Sandín (2003) y Palella y Martins, se puede destacar que, dependiendo del tipo de estudio el investigador puede plantear objetivos descritos en la Tabla 1.

\section{Tabla 1.}

Tipos de objetivos

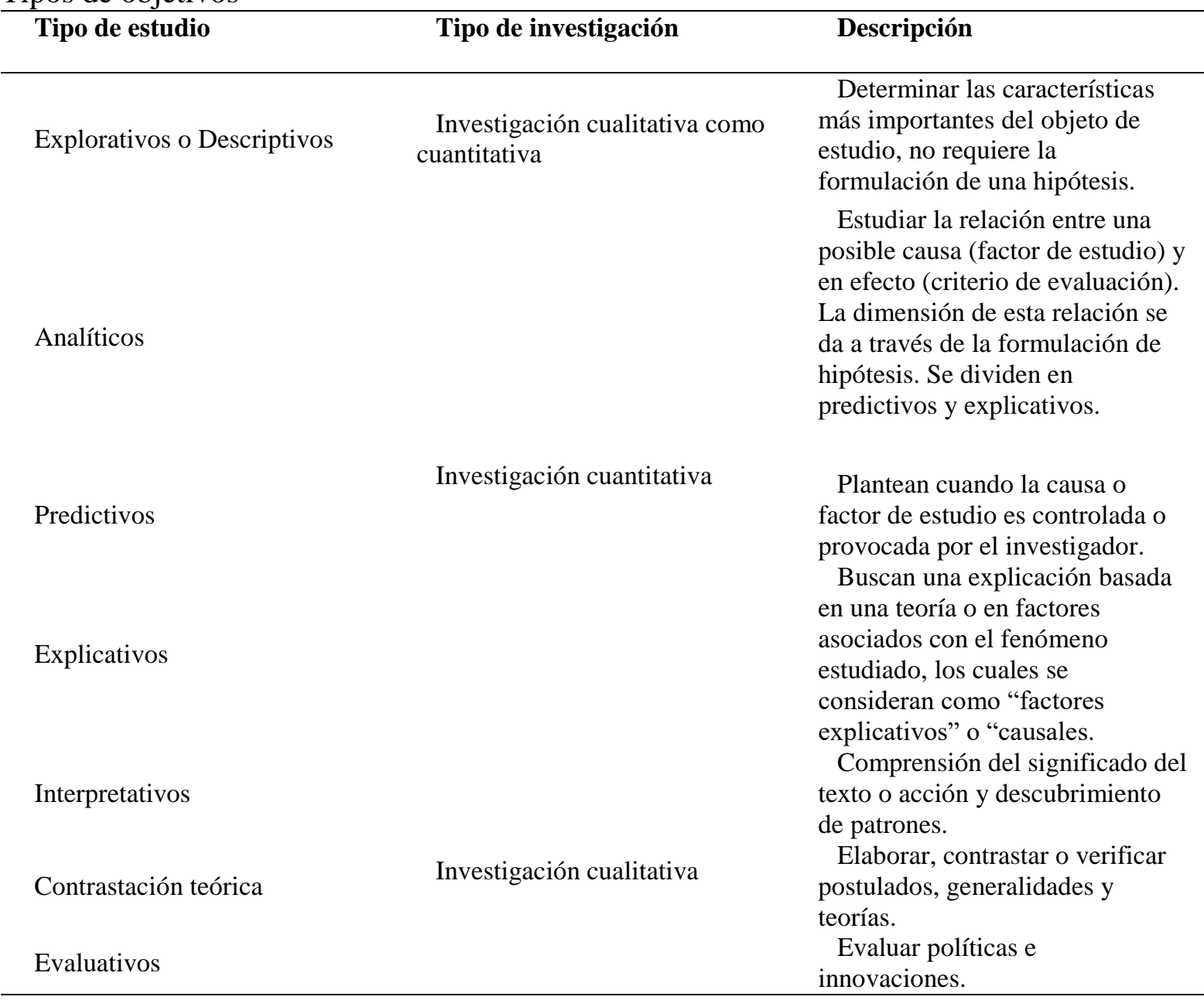

Fuente: elaboración propia.

La investigación cuantitativa, según Taylor y Bogdan (1986) hacen alusión a que "los positivistas buscan los hechos o causas de los fenómenos sociales con independencia de los estados subjetivos de los individuos", es decir pretenden buscar la objetividad. Este tipo de investigación los objetivos consisten en explicar las causas de los fenómenos, confrontar teoría y praxis, detectar discrepancias, analizar estadísticamente, establecer conexiones y generalizaciones (abstracciones).

Por su parte, los objetivos en la investigación cualitativa permiten describir e interpretar sensiblemente exacta la vida social y cultural de quienes participan. "El fenomenólogo quiere entender los fenómenos sociales desde la propia perspectiva del actor" (Taylor y Bogdan, 1986). La investigación cualitativa se centra en el significado, comprensión de la realidad, para ello se 
utilizan verbos que orienten a la exploración, la descripción, la comprensión, el descubrimiento de significados.

La Tabla 2 resume las características de los objetivos para la investigación cualitativa y cuantitativa.

\section{Tabla 2.}

Características de los objetivos de la investigación

\begin{tabular}{|c|c|}
\hline Tipo de investigación & Características de los objetivos \\
\hline Cuantitativa & 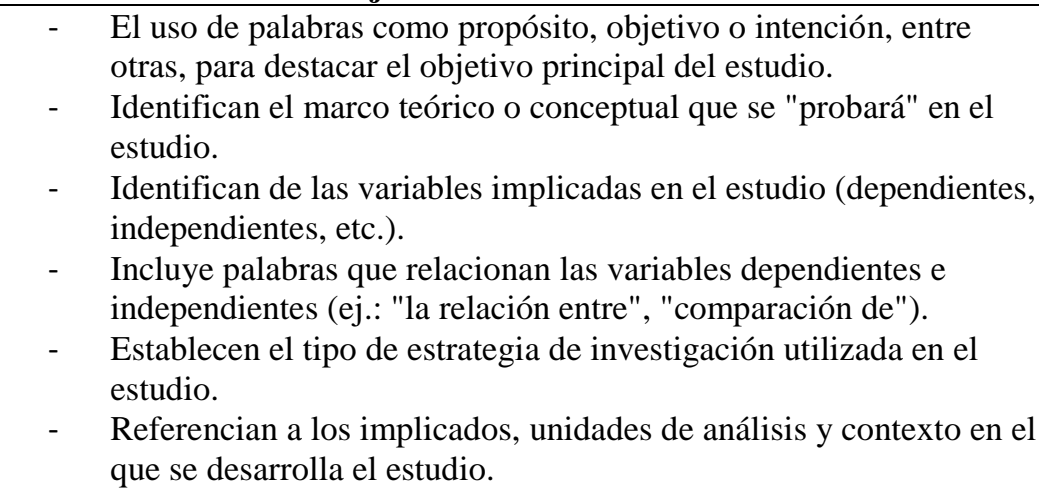 \\
\hline Cualitativa & $\begin{array}{l}\text { - } \begin{array}{l}\text { El uso de palabras como propósito, objetivo o intención, entre } \\
\text { otras, para destacar su importancia en la comprensión y desarrollo } \\
\text { del estudio. }\end{array} \\
\text { - } \quad \text { Se centran en un único fenómeno, concepto o idea. } \\
\text { - } \quad \text { En el caso de diseños emergentes, el empleo de palabras y frases } \\
\text { neutras, no directivas o prescriptivas. } \\
\text { - } \quad \text { Se proporcionan una definición inicial (no determinante) del } \\
\text { fenómeno objeto de estudio. } \\
\text { - Se mencionan los participantes en el estudio. } \\
\text { - Se comentan el contexto en el que se desarrollará la investigación. }\end{array}$ \\
\hline
\end{tabular}

Fuente: Adaptado de Taylor y Bogdan (1986)

\section{Escenario o contexto social}

El escenario o contexto social comprende la descripción del lugar donde se pretende llevar a cabo la investigación, ésta contendrá todos los aspectos que caracterizan el lugar seleccionado (ubicación geográfica, elementos físicos, aspectos poblacionales, condición socioeconómica general, entre otros), tal caracterización debe hacerse lo más objetivamente posible (Gurdián, 2007).

Con ello se aspira que, al describir cuál será el contexto actual en el cual se desarrollan los procesos investigativos a nivel socioeducativo, se tome en consideración los referentes de sentidos, situaciones y condiciones en las que se estructuran y constituyen las realidades sociales, así como también todo lo que acontece y sucede en un tiempo y espacio, lo que implica comprender y articular las distintas dimensiones de la realidad.

En este sentido, se plantea que el escenario constituye uno de los factores que influyen en el diseño de un proyecto de investigación asociados a los referentes (sociales, políticos, demográficos, sociales, económicos) que lo caracterizan en el contexto/ país del estudio, así 
como aquellos argumentos que propician su abordaje. Al respecto, Jiménez y Cols.(2006) esboza que es necesario delimitar dentro de un contexto teórico, disciplinar, tecnológico o profesional un objeto de indagación, una interrogante o un conjunto de interrogantes cuyo abordaje generará nuevos conocimientos o validará los existentes en nuevos contextos.

El escenario de estudio en la investigación cualitativa centra su atención en la experiencia humana, perfilando y teniendo lugar en contexto particulares, de manera que los acontecimientos y fenómenos no pueden ser comprendidos adecuadamente si son separados de aquellos. Por tanto, los contextos de investigación son naturales y no son construidos ni modificados, donde el investigador focaliza su atención en esos ambientes naturales a fin de dar respuesta a las cuestiones del mundo real (Sandín, 2003).

En particular, desde la investigación cualitativa el escenario o contexto de estudio se requiere para comprender los acontecimientos y conductas donde ocurren. Así, que las entidades sociales según Aravena y Col. (2006) y Sandín (2003) son concebidas como globalidades y holísticas que deben ser entendidas y explicadas en su integralidad. Esto da lugar a una concepción de la investigación donde el investigador debe desarrollar una sensitividad hacia situaciones o experiencias consideradas en su globalidad y hacia la cualidad que las regulan. Tomando en cuenta, los significados que las personas atribuyen a su conducta y a la de los demás, contextualizadas en el marco de los valores, prácticas y consiguientes estructuras de las que dichas personas y conductas forman parte.

Todo ello, con el propósito de interpretar o comprender el fenómeno social como un todo y el significado que ello tiene para sus participantes, a fin de basarse en el supuesto de que cualquiera sea el ámbito donde los datos sean recolectados, el investigador solamente podrá entender los acontecimientos si los sitúa en un contexto social e históricamente amplio.

Por otro lado, el ambiente en donde se realiza la investigación socioeducativa en el paradigma cuantitativo tiene aspectos "artificiales", es decir se recurre a escenarios en los cuales se pretende aislar y controlar situaciones intervinientes, abstraer una serie de variables, en donde el investigador y sujetos de estudio viven una especie de ambiente tipo laboratorio. Todo ello con la finalidad de que los resultados de la investigación se consolidan a través de las mediciones y predicciones exactas del comportamiento regular de grupos sociales.

Se debe definir el contexto en cuanto al tiempo y al espacio a través de la delimitación, para ello se especifican fronteras espacio-temporales dentro de las que se desarrollara la investigación, el cual comprende todo lo que abarcará como es a los individuos, las regiones geográficas y/o espaciales, lapso de tiempo o periodo del cual trata el estudio y todo aquellos elementos que limiten de alguna manera la generalización de los resultados a la totalidad de la población, como qué personas, materiales, situaciones, factores y causas serán consideradas o no (Sabino, 1986).

\section{Conclusiones}

A modo de cierre, conviene hacer énfasis en que todavía queda por definir algunos elementos organizacionales que constituyen la problemática de investigación, especialmente a 
nivel operacional. El investigador en muchas ocasiones ve la realidad socioeducativa como una parcela de un todo y busca dar respuestas a esa parcialidad, sin tomar en cuenta el universo de necesidades que el contexto originario e investigativo pudiera considerar; lo cual requiere de una visión inter y transdisciplinar para acoplarse a la amplitud de los problemas y descubrir el contexto social en forma global en la que circunscribe lo investigado.

La investigación educativa como un acto de investigación social comprende al ser humano como elemento partícipe de un proceso educativo dado su propia naturaleza que le permite circunscribirse en un contexto histórico determinado por el tiempo y el espacio. Sin embargo, quedan las diversas experiencias del hombre no limitadas al pasado, al presente y al futuro ni marcadas por las distancias, sino, como sustentos de horizontes de sentido para su existencia.

De igual manera, se destaca que se deben concretar la información que se tiene a la mano, las ideas claves y argumentos teóricos que permitan consolidar la detección de los nódulos críticos y aspectos macro, meso y micro que envuelven la inquietud impulsadora. Para ello, es necesario el re-educarse para ser capaz de re-pensar el mundo en que se vive, a fin de vincular dialécticamente la educación y la investigación con el contexto social en el que se desenvuelven. Todo ello, trae consigo que el conocimiento no sea ni neutral ni objetivo, sino que debe darse a partir de una construcción social, que encarne determinados intereses y supuestos.

Desde esta perspectiva, se concibe ontológicamente la realidad a investigar como una construcción social multidiversa, dinámica, tanto por los individuos participantes directamente en su contexto como por los investigadores que se dediquen a estudiarla. Por consiguiente, esta realidad compleja es relativamente desconocida para el investigador, es por ello que la autora fija una postura divergente a las posiciones metodológicas elaboradas a priori, donde se concibe el proceso de investigación social de manera lineal, más impacientadas por los procedimientos técnicos que por la diversidad y complejidad de la realidad a investigar.

\section{Bibliografía}

Aravena, M; Kimelman, E.; Micheli, B.; Torrealba, R. y Zúñiga, J. (2006). Investigación Educativa. Compilación Convenio Interinstitucional AFEFCE/Ecuador y Universidad Arcis/Chile. Disponible en: http://jrvargas.files.wordpress.com/2009/11/investigacioneducativa.pdf

Arellano, J y Santoyo, M. (2009). Investigar con mapas conceptuales: procesos metodológicos. Ediciones Narcea. Madrid. España.

Aristóteles _.. Metafísica. Escuela de Filosofía Universidad de ARCIS, edición en línea. Edición de Inmanuel Bekker (1831). Traducción de Valentín García Yebra. Disponible en: http://www.philosophia.cl/biblioteca/aristoteles/metafisica.pdf

Barrera, M. (2008). Modelos Epistémicos en Investigación y Educación. 5ta. Edición. Ediciones Quirón. Caracas.

Briones, G. (1996). Metodología de la Investigación cuantitativa en las ciencias sociales. Modulo 3. Instituto Colombiano para el fomento de la Educación Superior, ICFE. Disponible en: http://www.slideshare.net/adrysilvav/18-ok-ok-manual-investigacion-cientifica-enciencias-sociales 
Bunge, M. (1972). ¿Qué es el problema científico? Trabajo expuesto en Holmbergia, Revista del Centro de Estudiantes de Ciencias Naturales. Buenos Aires, Universidad de Buenos Aires. Pp. 1-14. Disponible en: http://inoctavo.com.ar/problema-cientifico-mario-bunge/

Carramolino, B. (2009). La revisión del Estado del Arte. Metodología de la Investigación. Valladolid. Disponible en: http://www.gsic.uva.es/wikis/yannis/images/3/36/Carramolino_Beatriz_MI.pdf

Collela, L. (2016). "Un análisis ontológico de la educación a partir de los aportes de Badiou y Rancière". Estudios de Filosofía, 53 (2016): 165-182.

De Melo, E. y Casanoves, F. (2010). Curso Ciencia y Sociedad: desafíos del milenio Escuela de Posgrado. Turrialba, Cossta Rica. Disponible en: http://intranet.catie.ac.cr/intranet/posgrado/InvestCienciaGestionConocimiento/Elias\%20 de\%20Melo\%20y\%20Fernando\%20Casanoves/Sesi\%C3\%B3n11_CiencSocied\%20$\%$ 20EdM-FCasan.pdf

Denman, C. y Haro J. (2002). Por los Rincones. Antologías de Métodos Cualitativos en la Investigación Social. El colegio de Sonora. Hermosillo, Sonora. pp.113 -145. Disponible en: http://psicologiaexperimental.files.wordpress.com/2010/02/

Ferrater, J. (2008) Diccionario de Filosofía Abreviado. 2da. Edición. Ediciones Debolsillo. Buenos Aires.

Gadamer, H. (1995). Hermenéutica y Diferencia Ontológica. Gessammelte Werke, Vol. 10. Pp.5870 En los Caminos de Heidegger. Traducción de Angela Ackermann. Disponible en: http://www.heideggeriana.com.ar/gadamer/hermeneutica_diferencia.htm

Gómez V., M., Galeano H., C. y Jaramillo M., D. A. (julio-diciembre, 2015). El estado del arte: una metodología de investigación. Revista Colombiana de Ciencias Sociales, 6(2), 423442

Guevara P., R. (2016). El estado del arte en la investigación: ¿análisis de los conocimientos acumulados o indagación por nuevos sentidos? FOLIOS. Segunda época. No 44 Segundo semestre de 2016. pp. 165-179. Recuperable de: http://www.scielo.org.co/pdf/folios/n44/n44a11.pdf

Gurdián, A. (2007). El Paradigma Cualitativo en la Investigación Socio-Educativa. Coordinación Educativa y Cultural Centroamericana CECC. San José, Costa Rica. Extraído el 23 de julio de 2012. Disponible en: http://ceccsica.org/programasaccion/educa/publicaciones_pdf/El_Paradigma_Cualitativo.pdf

Jiménez B., A.; Torres C., A.(comp.) (2006). La práctica investigativa en ciencias sociales. DCS, Departamento de Ciencias Sociales. UPN, Universidad Pedagógica Nacional, Bogotá, Colombia. ISBN: 958-8226-21-X. Disponible en: http://biblioteca.clacso.edu.ar/ar/libros/colombia/dcsupn/practica.pdf

Legrá L., A. y Silva D., O (sf.). La Investigación Científica: Conceptos y Reflexiones. Disponible en:

http://judithmetodologia.wikispaces.com/file/view/Libro+metodologia+La+Investigaci\% $\mathrm{C} 3 \% \mathrm{~B} 3 \mathrm{n}+\mathrm{Cient} \% \mathrm{C} 3 \%$ ADfica+CR+34b+Final+ISMM+(3).pdf

Maturana, H. (1997). La realidad: ¿objetiva o construida? Fundamentos Biológicos del Conocimiento, Tomo 2, Editorial Anthropos, Universidad Iberoamericana ITESO, Guadalajara, México.

Mella, O. (2007) Naturaleza y Orientaciones Teórico - Metodológicas de la Investigación Cualitativa. Guía Práctica de la Investigación Cualitativa-Cuantitativa. Revista Cándidus. pp. 81-89. 
Meza Cascante, L. G. (2013). Metodología de la investigación educativa: posibilidades de integración. Revista Comunicación, 182-194. https://doi.org/10.18845/rc.v12i1.1223

Morales Zúñiga, L. (2016). Los niveles de la investigación socioeducativa: lógica y relacionalidad investigativa. Revista Latinoamericana de Estudios Educativos (México), XLVI (4), 6596. Recuperable de: http://www.redalyc.org/pdf/270/27049500004.pdf

Padrón J. (1996). ¿Qué es un Problema de Investigación?. Extraído el 02 de Febrero, 2012 de http://www.uazuay.edu.ec/bibliotecas/Que\%20es\%20un\%20Problema\%20de\%20Investig acion.pdf

Palella S., S. y Martins P., F (2004). Metodología de la investigación cuantitativa. Fondo Editorial de la Universidad Pedagógica Experimental Libertador. Caracas, Venezuela.

Prévost, P. y Roy, M. (2007) La investigación sobre Gestión de Cooperativas: Algunas Reflexiones Fundamentales. Revista Unircoop Vol. 5. $\mathrm{N}^{\circ} 1$. pp.231-254. Disponible en: http://www.unircoop.org/unircoop/files/revue/Revue\%202007/11_prevost\%20et\%20roy. pdf

Rodríguez, D. y Valldeoriola, J. (2009) Metodología de la Investigación. Universidad Abierta de Cataluya. UOC. Extraído el 24 de julio de 2012. Disponible en: http://www.zanadoria.com/syllabi/m1019/mat_cast-nodef/PID_00148556-0.pdf

Ruedas, M., Ríos, M., Nieves, F. (2007). Epistemología de la Investigación Cualitativa. Extraído $\begin{array}{lllll} & 02 & \text { de } & \text { Febrero, } & 2012 \\ \end{array}$ http://www.saber.ula.ve/bitstream/123456789/31247/1/articulo7.pdf Sandoval, C. (1996). Especialización en Teoría, Métodos y Técnicas de Investigación Social. Investigación Cualitativa. Módulo 4. Universidad ICFES, Bogotá Colombia.

Sabino, C (1986). El proceso de investigación. Editorial Panapo, Caracas.

Sandín E., M. P (2003). Investigación Cualitativa en Educación. Fundamentos y Tradiciones. España. McGraw Hill Interamericana de España.

Taylor, S. J. y Bogdan, R. (1986). Introducción a los métodos cualitativos de investigación. La búsqueda de significados. Buenos Aires: Editorial Paidós. 